Int. J. Dev. Biol. 48: 623-627 (2004)

doi: $10.1387 / \mathrm{ijdb} .041887 \mathrm{rm}$

Original Article

\title{
Pbx genes are required in Xenopus lens development
}

\author{
RICHARD MORGAN* ${ }^{*}$, JASTINDER SOHAL ${ }^{1}$, MITA PALEJA ${ }^{1}$ and RUTH PETTENGELL ${ }^{2}$ \\ ${ }^{1}$ Department of Basic Medical Sciences, St. George's Hospital Medical School, London, U.K. and ${ }^{2}$ Department of Cellular and Molecular \\ Sciences, St. George's Hospital Medical School, London, UK.
}

\begin{abstract}
Development of the vertebrate eye is dependent on multiple tissue interactions and distinct sets of transcription factors and signalling molecules. The latter are thought to be FGF8 and BMP4, proteins secreted from the neural plate to induce lens formation in the overlying ectoderm. Here we show that the $\mathbf{P b x}$ transcription factor plays a crucial role in this process, as it is required for FGF8 expression in the neural plate and is thus part of the complex hierarchy of genes involved in lens induction.
\end{abstract}

KEY WORDS: Pbx, eye development, lens induction, FGF8, BMP, HOX

\section{Introduction}

The development of the vertebrate eye is one of the most extensively studied processes in embryology due to its accessibility in a number of different model systems. These studies have shown that eye development involves multiple tissue interactions that are regulated by a set of highly conserved developmental genes. The first of these interactions occur during gastrulation, when the involuting mesoderm and endoderm interact with the head ectoderm to give it a lens-forming bias (reviewed by Saha etal., 1989). Subsequently an extension of the diencephalon, the optic vesicle, induces the overlying head ectoderm to form a lens placode which then invaginates to form the lens. The optic vesicle gives rise to the optic cup, the outer layer of which ultimately differentiates into the pigmented retina, and the inner cell layer gives rise to the multitude of cell types that together form the neural retina.

The competence of the head ectoderm to form a lens is dependent upon the expression of the paired box / homeodomain containing transcription factor Pax6(Fujiwara etal., 1994; Li etal., 1994), and indeed, the ectopic expression of Pax6in the ectoderm of Xenopus embryos results in the formation of additional eye structures (Onuma et al., 2002). Pax6 expression is not required in the optic vesicle to induce lens formation in the overlying ectoderm though; this process depends instead on other genes that have not yet been fully characterised, but they include the secreted proteins BMP4 and FGF8. The former induces the expression of the Sox 2 and Sox 3 transcription factors in the overlying ectoderm, whilst FGF8 induces the expression of the LMaf gene (Furuta and Hogan, 1998; Ogino and Yasuda, 1998; Vogel-Hopker et al., 2000), and the combination of Pax6, Sox2, Sox3 and L-Maf ensures that the responding ectodermal cells differentiate into lens (Zygar et al., 1998).
In addition to BMP4 and FGF8, a number of transcription factors that have conserved functions in development are also expressed in the Xenopus forebrain, including $P b \times 1 b$ (Maeda et al., 2002). This is a homologue of the Drosophila extradenticle gene, a homeodomain-containing transcription factor. It can bind to a diverse set of other transcription factors as part of a heteromeric complex, including engrailed (Peltenburg and Murre, 1997), the myogenic factor TFS (Knoepfler etal., 1999), and the glucocorticoid receptor (Subramaniam et al., 2003). The most extensively characterised PBX interaction is with the HOX proteins, however. These are a family of homeodomain-containing transcription factors that determine cellular identity during development (reviewed by Carroll 1995; Gehring 1998; Burke 2000), and which also regulate cell proliferation and differentiation in the adult, most notably that of hematopoietic progenitor cells (Antonchuk et al., 2002; Kyba et al., 2002). The interaction between PBX and HOX / Engrailed proteins is dependent on a short stretch of highly conserved amino acids in the amino terminal of the HOX protein, known as the hexapeptide sequence (Phelan etal., 1995; Passner et al., 1999; Piper et al., 1999; Neuteboom et al., 1995). PBX binding to HOX proteins significantly increases their affinity and specificity of DNA binding (Chang et al., 1996).

The antisense-mediated depletion of $P b x$ genes in the primitive chordate Ciona results in severe developmental abnormalities (Yamada et al., 2003). A similar approach in Zebrafish that blocks the function of the Pbx2and Pbx4genes leads to a 'ground state' being imposed on the hindbrain, whereby segmentation is prevented and the entire structure takes on the identity of the first

Abbreviations used in this paper: BMP, bone morphogenetic protein; EDC, 1ethyl-3-[3-(dimethyl-amino) propyl] carbodiimide; FGF, fibroblast growth factor; Q-PCR, quantitative Polymerase Chain Reaction.

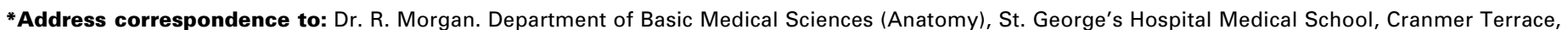
London SW17 0RE, U.K. Fax: +44-208-725-3326. E-mail: rmorgan@sghms.ac.uk
} 
rhombomere (Waskiewicz et al., 2002; Cooper et al., 2003). Blocking Pbx 1 bactivity in Xenopusalso results in neural defects, whereby the induction of gene expression associated with the posterior neural tube and the neural crest is prevented (Maeda et al., 2002).

We have used a novel strategy for blocking PBX binding to its cofactors to inactivate it in the isolated neural plate from Xenopus embryos. This in turn prevents the expression of FGF8, and blocks lens induction in adjacent head ectoderm explants.

\section{Results and Discussion}

Pbx genes are expressed in distinct tissues of the developing embryo, and the phenotype associated with a general ablation of $P b x$ activity reflects this. A different approach is therefore required to study the role of $P b x$ in a specific tissue, especially at a relatively late stage of development. For this reason, we developed a strategy for blocking the binding of PBX protein to its cofactors, taking the highly conserved HOX hexapeptide sequence WYPWMKKHH - which is known to mediate this process (reviewed by Morgan et al., 2000) - and making a synthetic peptide containing both it and a 16 amino acid sequence ('penetratin') based on the Drosophila Antennapedia protein, previously shown to mediate efficient movement of proteins across cell membranes (Derossi et al., 1998). This peptide is referred to as HXP4. In addition, a control peptide was made consisting of a non-hexapeptide sequence (WCPWLDRHGR) linked to penetratin (CXP4). The ability of HXP4 to prevent HOX / PBX interactions was tested by adding it to anterior neural plate isolated from neurula stage Xenopusembryos. Total cell protein was then analysed for PBX by western blotting (Monica et al., 1991), with or without cross linking (Fig. 1). HXP4, but not the control peptide CXP4, prevented binding of PBX to other

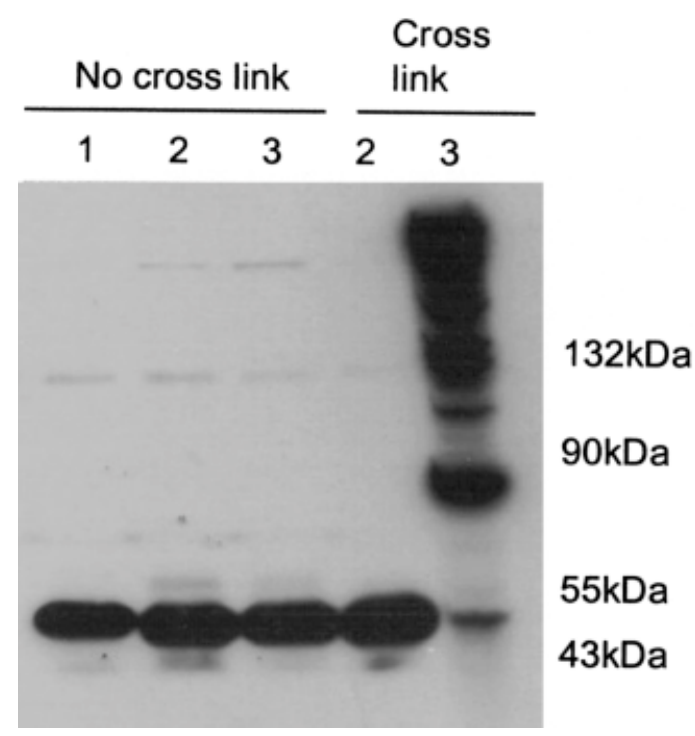

Fig. 1. Disruption of HOX and PBX binding by HXP4. Western blot of protein extracted from the dorsal ectoderm of neurula stage Xenopus embryos and probed with an antibody raised against PBX1,2 and 3 isoforms. 'Cross-linked' - protein was extracted in 1-ethyl-3-[3-(dimethylamino)propyl]carbodiimide (EDC) to cross link non-covalently associated proteins. (1) Untreated ectoderm; (2) CXP4 peptide-treated ectoderm; (3) HXP4 peptide-treated ectoderm. The presence of HXP4 disrupted the formation of PBX / HOX dimers from PBX monomers (52 kDa).

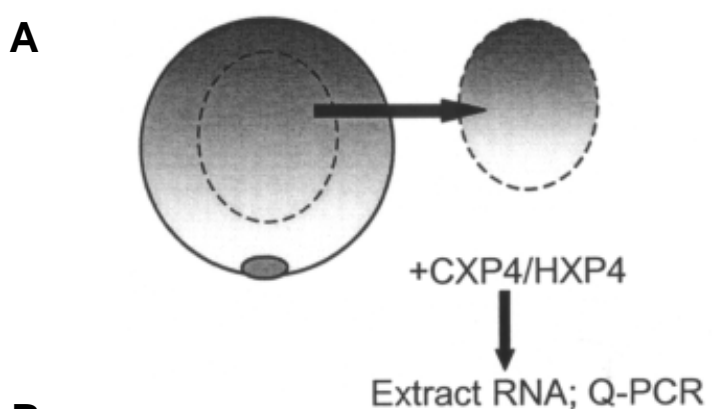

B

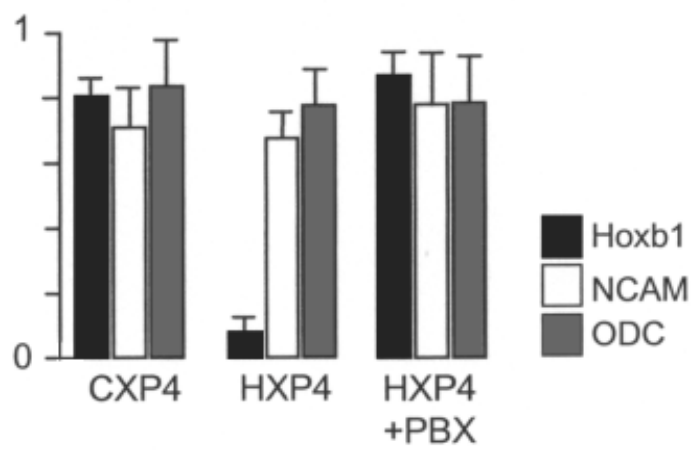

Fig. 2. HXP4 inhibits Hoxb1 expression in the neural plate. (A) The neural plate was isolated from early neurula (stage 12.5) Xenopus embryos and incubated with HXP4 or CXP4, as shown. Subsequently RNA was extracted for Q-PCR analysis. (B) The relative expression levels of Hoxb1, NCAM and ODC as determined by Q-PCR, standardised to that of ef1alpha. The amount of each transcript is shown relative to that in untreated, control neural plates. The error bars represent the SEM from three experiments.

proteins under these conditions. Hence HXP4 can act as a competitive inhibitor of $\mathrm{PBX}$ function by blocking its interaction with other co-factors. In order to test that HXP4 does indeed have this property, its affect on the expression of Hoxb1 in the neural plate was assessed. Hoxb1 expression is maintained in this tissue by an autoregulatory loop that is dependent on an interaction between HOXB1 and PBX proteins (Di Rocco et al., 1997). Isolated neural plates excised from early neural (stage 12.5) embryos were incubated with HXP4 or CXP4 for two hours, whereupon the total RNA was extracted and assayed for the expression of Hoxb1 and NCAM (which is expressed in both Hoxb1-expressing and nonexpressing neural cells). Treatment with HXP4, but not CXP4, resulted in a significant decrease in the expression of Hoxb1, whilst neither peptide affected the expression of $N C A M$ (Fig. 2). Furthermore, neural plates taken from Pbxover-expressing embryos did not show any reduction in Hoxb1 expression (Fig. 2). Hence HXP4 is a specific inhibitor of PBX interactions.

In order to further characterise the activity of HXP4, we also examined the expression of a reporter gene driven by a previously characterised HOXB4 / PBX responsive element (Morsi El-Kadi et al., 2002; White et al., 2000). This was microinjected into fertilised eggs together with Hoxb4RNA, and naive ectoderm was subsequently removed from these embryos at the late gastrula stage (12.5). In the presence of HOXB4 the reporter construct shows a high level of activity, which is abolished if an altered HOXB4 / PBX consensus site is used. CXP4 has no affect on reporter activity, whilst HXP4 causes a significant reduction (Fig. 3). Furthermore, the co-injection 
A
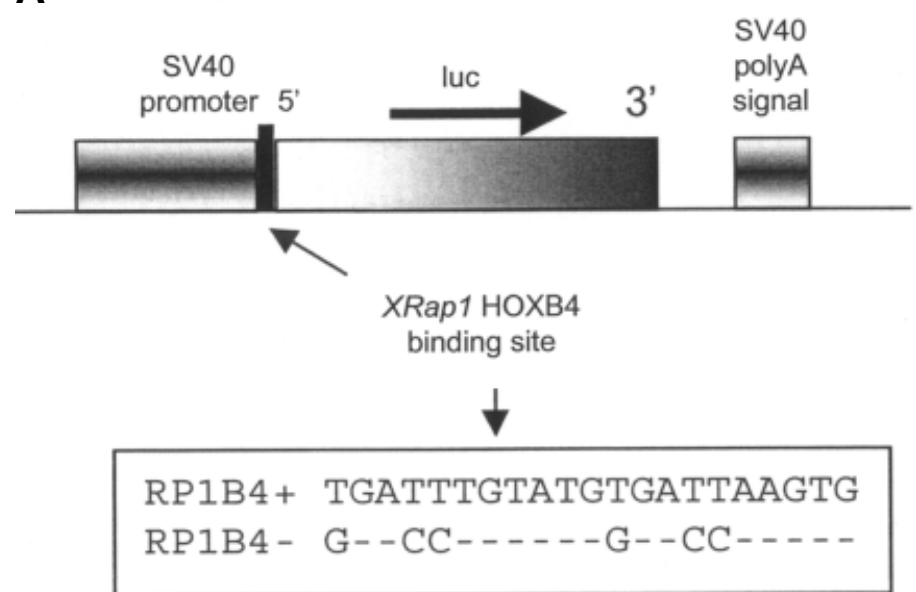

B

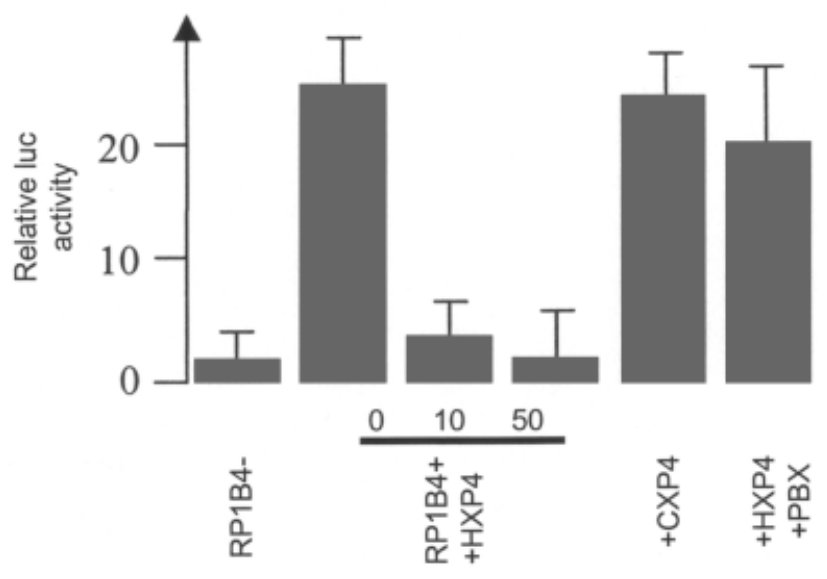

Fig. 3. HXP4 blocks the Hoxb4-activated transcription of a reporter construct. (A) The reporter constructs were based on the $p G L 3$ vector which contains the luc gene under the control of the ubiquitously active SV4O promoter. The putative HOXB4 binding site from XRap1 was cloned immediately $5^{\prime}$ to the luc gene, as shown. The nucleotide sequence of the putative HOXB4 binding region (RP1B4+) and a non-binding variant used as a control (RP1B4-) are shown. (B) Explants derived from RP1B4+ injected embryos were treated with 0,10 or 50 microgrammes/ml of HXP4 for 12 hours, after which time luciferase activity was measured. Alternatively, explants were treated with $50 \mu \mathrm{g} / \mathrm{m} / \mathrm{CXP} 4(+C X P 4)$. As an additional control, embryos were injected with both RP1B4+ and $1 \mathrm{ng} \mathrm{Pbx} R N A$, and excised explants were treated with $50 \mu \mathrm{g} / \mathrm{ml}$ HXP4 (+HXP4+PBX) for 12 hours.

of $P b x$ into embryos rescues reporter activity, indicating that the effects of HXP4 are specific for protein interactions involving the hexapeptide sequence.

The optic vesicle is derived from the anterior neural plate, and in Xenopus the anterior neural plate alone is sufficient to induce the expression of lens specific genes in naïve ectoderm (Zygar et al., 1998). One of the genes expressed in the anterior neural plate is $P b \times 1 b$ (Maeda et al., 2002), and we decided to use HXP4 to test whether it is required for lens induction. Anterior neural plate was removed from mid neurula (stage 14) embryos and cultured for three hours in either high salt buffer (MMR) alone, MMR with CXP4, or MMR with HXP4 (Fig. 4A). After this time the total RNA was extracted from the explants and analysed for the expression of a number of different genes using quantitative - PCR (Q-PCR). HXP4 treatment caused a significant decrease in the number of FGF8 transcripts, whilst the expression of both BMP4 and the housekeeping gene ornithine decarboxylase (ODC) was unaffected (Fig. 4B). Hence of the two previously identified lensinducing factors, only FGFBis dependent on Pbxfor its expression.
We made tissue recombinants of ectoderm removed from the animal pole of mid-gastrula (stage 11.5) embryos, and the anterior neural plate taken from neurual (stage 14) embryos which had been treated with HXP4 or CXP4 (Fig. 5A). These recombinants were cultured until their untreated sibling embryo had reached the late neurula (stage 21). Recombinants incubated without peptide or with the control peptide (CXP4) both expressed L-Mafand the lens-specific gene gamma-crystallin, whilst the HXP4 treated recombinants did not (Fig. 5B).

In order to further characterise gene expression in the lenscompetent ectoderm, RNA was also extracted from ectoderm removed when sibling embryos had reached the mid-neurala stage, and the relative amounts of $O D C$, Pax 6 , Sox2, Sox3and L-Marwere assayed by QPCR (Fig. 5C). This revealed a significant reduction only in $\angle$-Maf expression (Fig. 5C), a finding that concurs with previous studies indicating that $F G F B$ is required for the induction of $\angle-M a f e x p r e s s i o n$ in competent lens ectoderm (Ogino and Yasuda, 1998; VogelHopker et al., 2000), whilst BMP4 is required to induce Sox2 and Sox3expression (Furuta and Hogan, 1998).
A

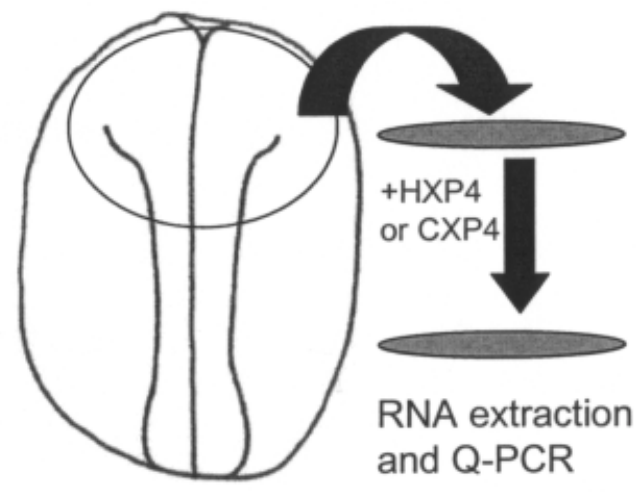

B

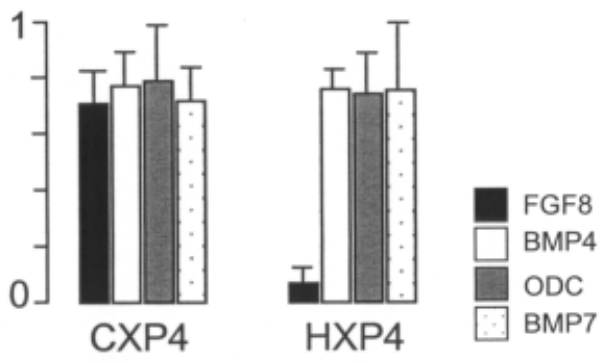

Fig. 4. HXP4 blocks the expression of FGF8 in the anterior neural plate. (A) The anterior neural plate was excised from neurula (stage 14) embryos and treated with HXP4 or CXP4, as shown. (B) The relative expression levels of FGF8, BMP4 and ODC were determined by $Q$ $P C R$, and standardised to that of ef1alpha. Values are shown relative to the untreated control. The error bars represent the SEM from three experiments. 
A

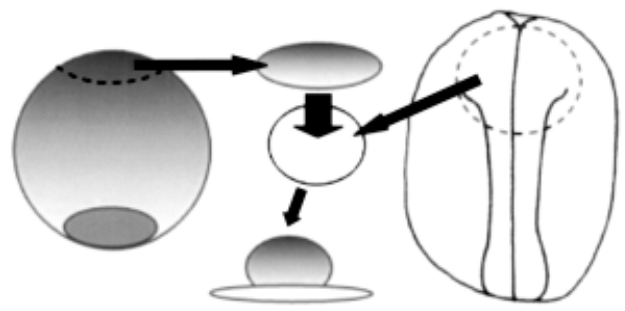

B

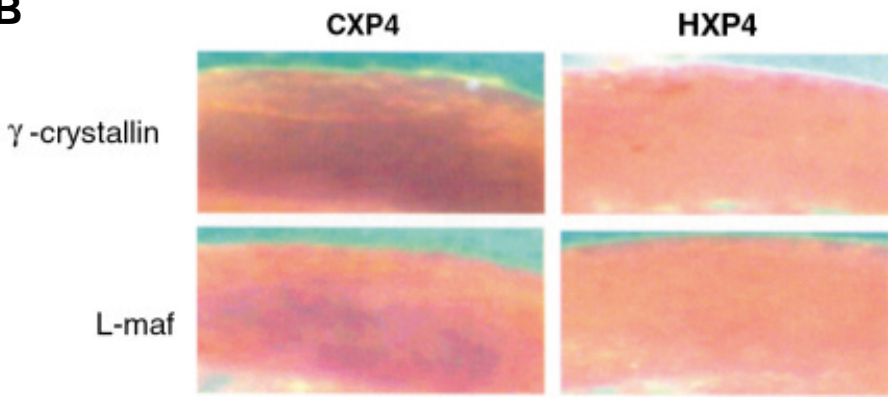

C

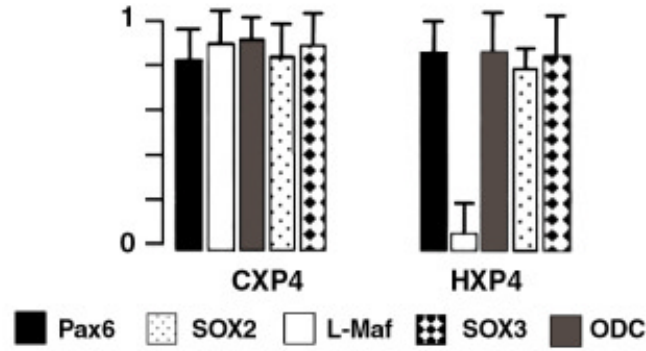

Fig. 5. HXP4 treated neural plate cannot induce lens formation in ectodermal explants. (A) Conjugates were made between ectoderm taken from mid gastrula (stage 11.5) embryos (left) and the anterior neural plate from neurula (stage 14) embryos. These were cultured until the sibling embryos had reached stage 21 (late neurula). (B) The explants were analysed by in situ hybridisation for gamma-crystalline and L-Maf expression (purple staining). (C) The explants were also analysed for Pax6, L-Maf, ODC, Sox2 and Sox3 expression by Q-PCR. The amount of each transcript is shown relative to that in untreated, control neural plates. The error bars represent the SEM from three experiments.

The requirement for Pbxin FGF8expression suggests that this transcription factor and at least some of its cofactors are in the hierarchy of genes that regulate lens induction. This hierarchy involves distinct sets of transcription factors and signalling molecules, and two distinct tissues. In the anterior neural plate (including those cells that form the optic vesicle), $P b x$ activates the expression of $F G F 8$, and this in turn activates expression of the L-Maftranscription factor in the overlying ectoderm (Ogino and Yasuda, 1998; Vogel-Hopker et al., 2000). Other, as yet unidentified transcription factors drive the expression of BMP4 in the neural plate, and this signalling molecule in turn activates the expression of Sox2 and Sox3 in the lens-competent ectoderm (Furuta and Hogan, 1998).

In addition to lens induction, FGF8 also functions within the neural plate to pattern the developing brain, as its release from the midbrain / hindbrain boundary is required for the expression of En2 and $W n t 1$ in the posterior midbrain (Crossley et al., 1996; Riou et al., 1998). The expression of Pbxis considerably wider than that of
FGF8, as it is expressed throughout the neural plate and is not confined to the midbrain / hindbrain boundary. Presumably the greater specificity of FGF8 expression is regulated by specific $P b x$ co-factors, a likely candidate being engrailed 2, which is coexpressed with FGF8. This is supported by the presence of EN2 / $\mathrm{PBX}$ binding sites within an intronic enhancer of the FGF8 gene, which are necessary to maintain FGF8 expression in ES cells (Gemel et al., 1999). It is likely that further work will help to identify additional components of these complex regulatory pathways.

\section{Materials and Methods}

\section{RNA extraction and RT-QPCR}

Total RNA was extracted from whole embryos, isolated neural plate, or ectoderm using an RNeasy mini kit (Qiagen). One $\mu \mathrm{g}$ of RNA was used in subsequent reverse transcription reactions. This was mixed with a poly T15 oligo to $5 \mu \mathrm{g} / \mathrm{ml}$ and heated to $75^{\circ} \mathrm{C}$ for 5 minutes. After cooling on ice, the following additional reagents were added; dNTPs to $0.4 \mathrm{mM}$, RNase OUT (Promega) to $1.6 \mathrm{U} / \mu \mathrm{l}$, Moloney Murine Leukemia Virus Reverse Transcriptase (M-MLRvT) RnaseH- point mutant (Promega) to $8 \mathrm{U} / \mu \mathrm{l}$ and the appropriate buffer (supplied by the manufacturer) to $\times 1$ concentration. The mixture was incubated for one hour at $37^{\circ} \mathrm{C}$ heated to $70^{\circ} \mathrm{C}$ for two minutes and cooled on ice.

QPCR reactions were all performed in a total volume of $50 \mu \mathrm{l}$. For each we used $1 \mu \mathrm{l}$ of the M-MLRvT reaction (as described above), $0.2 \mathrm{nmol}$ of each primer and $25 \mu \mathrm{l}$ of pre-mixed QPCR components (Sigma). All reactions were cycled at $94^{\circ} \mathrm{C}$ for 30 seconds, $55^{\circ} \mathrm{C}$ for 30 seconds and $72^{\circ} \mathrm{C}$ for 60 seconds, for 45 cycles. The primers used for FGF8 amplification were;

FGF8 (5'): 5' ACC TCC ATC CTG GGC TAT CT 3' and

FGF8 (3'): 5' CAC GAT TAA CTT GGC GTG TG 3';

GCT (5'): 5' GTG GAG AGT GGC AAC TGG AT 3' and GCT(3'): 5' GTT GCC ATC AAA CAC ATT GC 3';

BMP4 (5'): 5' CAA CAC ACT TTT AAA ATG CTT ACG 3' and BMP4 (3'): 5' GGC TGG AAT GAA TGG ATT GT 3'; SOX2(5'): 5' TCT GCA CAT GAA GGA GCA TC 3' and SOX2 (3'): 5' CAC TTA TGC CCA CAT GAA CG 3'; SOX3 (5'): 5' ATG AAC GGC TGG ACT AAT GG 3' and SOX3(3'): 5' GGA GCT CTG CTG GTT GTA GG 3'; L-Maf(5'): 5' ACT CAG GGC TGT CCC CTA AT 3' and L-Maf(3'): 5' TTG GGG ATT TTG AGA CTT GC 3';

Pax6 (5'): 5' GCC ACA TTC CCA TTA GCA GT 3' and Pax6 (3'): 5' AGG TAG ATT GTT GCC CAT CG 3'.

The sequences of the other primer pairs can be found on the internet at: http://www.sghms.ac.uk/depts/anatomy/pages/richhmpg.htm/.

QPCR was performed using the SYBR green labelling kit from Sigma., using ROX as the internal standard dye. Thermal cycling and fluorescence detection was by a MX4000 (Stratagene Inc., USA). Semi-quantitative data was obtained by using measurements three cycles after reactions had risen above the base line, and where clearly in exponential increase. Ef1alpha was used as a loading control, and all values are presented as a ratio of target to ef1alpha signal.

\section{Whole mount in situ hybridisation of ectodermal conjugates}

The L-Maf reading frame was cloned into vector pGEMT-easy (Promega), and this was linearised using Ncol. A DIG-labelled in situ probe was transcribed from this template using SP6 polymerase. A DIGlabelled gamma-crystallin probe was transcribed as previously described (Godsave et al., 1994). Probe purification and subsequent in situanalysis were performed as described (Sive et al., 2000).

\section{Western blotting of PBX and PBX / HOX dimers}

Protein was extracted from HXP4-treated or CXP4 -treated $(20 \mu \mathrm{g} / \mathrm{ml})$ neural explants taken from stage 14 embryos. C-20 antibody recognises 
the human PBX proteins and cross - reacts with Xenopus PBX $1 B$ protein (sc-888, Santa Cruz Biotechnology Inc., USA). Protein cross linking was performed with $2 \mathrm{mM}$ 1-ethyl-3-[3-(dimethylamino)propyl]carbodiimide (EDC) (Pierce Biotechnology, USA), in cell lysates diluted 1:10 in conjugation buffer $(0.1 \mathrm{M} 2$-[N-morpholino] ethane sulphonic acid, $\mathrm{pH} 5)$. After 15 minutes at room temperature the reaction was stopped by adding 2-mercaptoethanol to $20 \mathrm{mM}$ and excess reagents were removed using a D-Salt Dextran desalting column (Pierce, USA).

\section{Acknowledgements}

The QPCR studies reported here were performed with the help and facilities of the Medical Biomics Centre at St. George's Hospital Medical School.

\section{References}

ANTONCHUK, J., SAUVAGEAU, G. and HUMPHRIES, R.K. (2002). HOXB4 induced expansion of adult hematopoietic stem cells ex vivo. Cel/109:39-45.

BURKE, A.C. (2000). Hox genes and the global patterning of the somitic mesoderm. Curr. Top. Dev. Biol. 47: 155-181.

CARROLL, S.B. (1995). Homeotic genes and the evolution of arthropods and cordates. Nature 376: 479-485

CHANG, C.P., BROCCHIERI, L., SHEN, W.F., LARGMAN, C. and CLEARY, M.L. (1996). PBX modulation of HOX homeodomain amino-terminal arms establishes different DNA-binding specificities across the HOX locus. Mol. Cell Biol. 16:17341745.

COOPER, K.L., LEISENRING, W.M. and MOENS, C.B. (2003). Autonomous and nonautonomous functions for $\mathrm{Hox} / \mathrm{Pbx}$ in branchiomotor neuron development. Dev. Biol. 253: 200-213.

CROSSLEY, P. H., MARTINEZ, S. and MARTIN, G. R. (1996). Midbrain development induced by FGF8 in the chick embryo. Nature 380: 66-68.

DEROSSI, D., CHASSAING, G. and PROCHIANTZ, A. (1998). Trojan peptides: the penetratin system for intracellular delivery. Trends Cell Biol. 8:84-87.

DI ROCCO, G., MAVILIO, F. and ZAPPAVIGNA, V. (1997). Functional dissection of a transcriptionally active, target-specific Hox-Pbx complex. EMBO J. 16: 3644-3654

FUJIWARA, M., UCHIDA, T., OSUMI-YAMASHITA, N. and UCHIDA, E.K (1994). Rat (rSey): a new mutant rat with craniofacial abnormalities resembling those of the mouse Sey mutant. Differentiation 57: 31-38.

FURUTA, Y. and HOGAN, B.L. (1998). BMP4 is essential for lens induction in the mouse embryo. Genes Dev. 12: 3764-3775.

GEHRING, W.J. (1988). Master control genes in development and evolution: the homeobox story. Yale University Press, New Haven and London.

GEMEL, J., JACOBSEN, C. and MACARTHUR, C.A. (1999). Fibroblast growth factor-8 expression is regulated by intronic engrailed and Pbx1-binding sites. J. Biol. Chem. 274: 6020-6026.

KNOEPFLER, P.S., BERGSTROM, D.A., UETSUKI, T., DAC-KORYTKO, I., SUN, Y.H., WRIGHT, W.E., TAPSCOTT, S.J. and KAMPS, M.P. (1999). A conserved motif $\mathrm{N}$-terminal to the DNA-binding domains of myogenic bHLH transcription factors mediates cooperative DNA binding with pbx-Meis1/Prep1. Nucleic Acids Res. 27: 3752-3761.

KYBA, M., PERLINGEIRO, R.C. and DALEY, G.Q. (2002). Hoxb4confers definitive lymphoid-myeloid engraftment potential on embryonic stem cell and yolk sac hematopoietic progenitors. Cel/109: 29-37.

LI, H.S., YANG, J.M., JACOBSON, R.D., PASKO, D. and SUNDIN, O. (1994). Pax6 is first expressed in a region of ectoderm anterior to the early neural plate: implications for stepwise determination of the lens. Dev. Biol. 162: 181-194.
MAEDA, R., ISHIMURA, A., MOOD, K., PARK, E.K., BUCHBERG, A.M. and DAAR, I.O. (2002). XPbX1b and Xmeis1b play a collaborative role in hindbrain and neural crest gene expression in Xenopus embryos. Proc. Natt. Acad. Sci. USA 99: 5448-5453.

MONICA, K., GALI, L.I., NOURSE, J., SALTMAN, D. and CLEARY, M.L. (1991). PBX2 and PBX3, new homeobox genes with extensive homology to the human proto-oncogene PBX1. Mol. Cell Biol. 11: 6149-6157.

MORGAN, R., IN DER RIEDEN, P., HOOIVELD, M.H. and DURSTON, A.J. (2000). Identifying HOX paralog groups by the PBX-binding region. Trends. Genet. 16: 66-67.

MORSI EL-KADI, A.S., IN DER REIDEN, P., DURSTON, A. and MORGAN, R. (2002). The small GTPase Rap1 is an immediate downstream target for Hoxb4 transcriptional regulation. Mech Dev113: 131-139.

NEUTEBOOM, S.T., PELTENBURG, L.T., VAN DIJK, M.A. and MURRE, C. (1995) The hexapeptide LFPWMR in Hoxb-8 is required for cooperative DNA binding with Pbx1 and Pbx2 proteins. Proc. Natl. Acad. Sci. USA. 92: 9166-9170.

OGINO, H. and YASUDA, K. (1998). Induction of lens differentiation by activation of a bZIP transcription factor, L-Maf. Science 280: 115-118.

ONUMA, Y., TAKAHASHI, S., ASASHIMA, M., KURATA, S. and GEHRING, W.J. (2002). Conservation of $\operatorname{Pax} 6$ function and upstream activation by Notch signaling in eye development of frogs and flies. Proc. Natl. Acad. Sci. USA 99: 2020-2025.

PASSNER, J.M., RYOO, H.D., SHEN, L., MANN, R.S. and AGGARWAL, A.K. (1999). Structure of a DNA-bound Ultrabithorax-Extradenticle homeodomain complex. Nature 397: 714-719.

PELTENBURG, L.T. and MURRE, C. (1997). Specific residues in the Pbx homeodomain differentially modulate the DNA-binding activity of Hox and Engrailed proteins. Development 124: 1089-1098.

PHELAN, M.L., RAMBALDI, I. and FEATHERSTONE, M.S. (1995). Cooperative interactions between HOX and PBX proteins mediated by a conserved peptide motif. Mol. Cell Biol. 15: 3989-3997.

PIPER, D.E., BATCHELOR, A.H., CHANG, C.P., CLEARY, M.L. and WOLBERGER, C. (1999). Structure of a HOXB1-PBX1 heterodimer bound to DNA: role of the hexapeptide and a fourth homeodomain helix in complex formation. Cel/ 96: 587-597.

RIOU, J.F., DELARUE, M., MENDEZ, A.P. and BOUCAUT, J.C. (1998). Role of fibroblast growth factor during early midbrain development in Xenopus. Mech. Dev. 78: 3-15.

SAHA, M.S., SPANN, C.L. and GRAINGER, R.M. (1989). Embryonic lens induction: more than meets the optic vesicle. Cell Differ. Dev. 28: 153-171.

SUBRAMANIAM, N., CAMPION, J., RAFTER, I. and OKRET, S. (2003). Cross-talk between glucocorticoid and retinoic acid signals involving glucocorticoid receptor interaction with the homeodomain protein Pbx1. Biochem. J. 370: 1087-1095.

VOGEL-HOPKER, A., MOMOSE, T., ROHRER, H., YASUDA, K., ISHIHARA, L. and RAPAPORT, D.H. (2000). Multiple functions of fibroblast growth factor-8 (FGF-8) in chick eye development. Mech. Dev. 94: 25-36.

WASKIEWICZ, A.J., RIKHOF, H.A. and MOENS, C.B. (2002). Eliminating zebrafish pbx proteins reveals a hindbrain ground state. Dev. Cel/3: 723-733.

WHITE, R.A., ASPLAND, S.E., BROOKMAN, J.J., CLAYTON, L. and SPROAT, G. (2000). The design and analysis of a homeotic response element. Mech. Dev. 91: 217-226.

YAMADA, L., SHOGUCHI, E., WADA, S., KOBAYASHI, K., MOCHIZUKI, Y., SATOU, Y. and SATOH, N. (2003). Morpholino-based gene knockdown screen of novel genes with developmental function in Ciona intestinalis. Development 130: 6485-6495.

ZYGAR, C.A., COOK, T.L. and GRAINGER, R.M. JR. (1998). Gene activation during early stages of lens induction in Xenopus. Development 125: 3509-3519.

Received: April 2004 Reviewed by Referees: May 2004 Modified by Authors and Accepted for Publication: June 2004 Edited by: Christine Mummery 